\title{
THREE-WEEK INTENSIVE NEUROMUSCULAR TRAINING IMPROVES POSTURAL CONTROL IN PROFESSIONAL MALE SOCCER PLAYERS
}

\author{
MAŁGORZATA KOWALCZYK ${ }^{1,2}$, PAWEŁ TOMASZEWSKIㄴ, NATALIA BARTOSZEK², \\ MARCIN POPIELUCH ${ }^{3}$ \\ Józef Pitsudski University of Physical Education, Faculty of Physical Education, \\ Department of Biometry, Warsaw, Poland \\ ${ }^{2}$ Carolina Medical Center, Warsaw, Poland \\ ${ }^{3}$ Polish Football Association, Warsaw, Poland
}

\author{
Mailing address: Małgorzata Kowalczyk, Józef Piłsudski University of Physical Education, \\ Faculty of Physical Education, Department of Biometry, 34 Marymoncka Street, 00-968 Warsaw, \\ tel.: +48 88 1412386, e-mail: kosim.malgorzata@gmail.com
}

\begin{abstract}
Introduction. The purpose of this study was to determine the impact of a short-term, three-week intensive neuromuscular training programme on the postural stability of professional soccer players and to establish whether lower-limb dominance affects one-leg stability. Material and methods. The experimental group consisted of 16 professional male soccer players, and the control group included 20 professional soccer players who did not undergo any additional training. The experimental group was provided with three-stage progressive training twice a day over a three-week period during the preparatory period and then trained twice per week in-season for a period of 5 months. The experimental subjects were evaluated with the use of a stabilographic platform before the programme, 3 weeks into the programme, and finally after 5 months from its commencement. Total centre of pressure (COP) sway path was measured during one leg stance for the dominant and non-dominant leg with eyes open and closed. Results. After three weeks of training, the experimental group showed a significantly shorter COP path for the measurements taken with both open $\left(p<0.001 ; \eta^{2}=0.28\right)$ and closed eyes $\left(p<0.05 ; \eta^{2}=0.09\right)$ compared to the initial results. It was found that the training effects were sustained five months into the training programme for the tests with eyes open $(\mathrm{p}<0.001)$ and eyes closed $(\mathrm{p}<0.05)$. The control group, however, showed no such improvement during that time. No differences were found between the limbs in either of the groups. Conclusions. Neuromuscular training can be effective for postural control improvement in professional male soccer players within a short period of time. Leg dominance does not affect one-leg stability.
\end{abstract}

Key words: proprioception, balance, injury prevention, training

\section{Introduction}

Soccer is a discipline requiring excellent postural control, which directly affects the level of sporting performance $[1,2]$ as well as susceptibility to injuries $[3,4,5]$. The available research results indicate that adequate neuromuscular training increases players' postural control and consequently reduces the occurrence of injuries $[6,7,8]$ such as ankle sprains [9-12], anterior cruciate ligament injuries $[6,13,14]$, and hamstring strains [12, $15,16,17]$. The effectiveness of such programmes in enhancing functional performance after a sports injury has also been demonstrated by several authors $[8,18,19,20]$. It has been suggested that such effects are predominantly linked with changes in proprioception and neuromuscular control [21].

However, despite the growing awareness of the impact of such training programmes on potential effects in soccer athletes, they still have not been widely incorporated into training sessions [22]. Based on the available literature, it has not been defined how such programmes should be constructed to optimise their time and efficacy. Most up-to-date prevention programmes last 6 to 8 weeks even though data concerning their optimal length are hardly sufficient to opt for the standardised option [23]. Zech et al. [7] found in a systematic review that pro- grammes lasting 6 weeks and longer had greater effect on postural sway than 4 -week programmes.

The authors of this article believe that soccer players, due to the specificity of the discipline, are required to develop better postural control in a time shorter than 6 weeks. For that reason, we created a programme which was shorter ( 3 weeks long) and more intense than most of the existing ones. The training protocol included exercises focusing on stability, agility, coordination, and plyometrics and introduced a number of varied activities constantly challenging the players' balance [24]. Another issue worth investigating is whether soccer players show differences in stability between their limbs and whether training should differentiate between the dominant and non-dominant limbs.

Having considered all these aspects, the authors decided to conduct a study whose purpose was to determine the impact of a short-term, 3-week intensive neuromuscular training programme on the postural stability of professional soccer players and to establish whether lower-limb dominance affects one-leg stability. The hypothesis put forward by the researchers was that such intensive training would significantly improve the postural control of professional soccer players. We also hypothesised that the non-dominant leg would show an improved level of stability compared to the dominant (kicking) leg. 


\section{Material and methods}

\section{Subjects}

A total of 36 top professional male soccer players (members of an elite soccer team) volunteered to participate in this study. The experimental group consisted of 16 players, and the control group included 20 subjects. Descriptive characteristics of the participants (mean, SD, max, and min), randomised into the experimental or control group, are provided in Table 1. The experimental participants suffered no injuries eliminating them from the game for more than 20 days during the previous 3 years. Additionally, the participants had to be free from acute musculoskeletal injuries and chronic musculoskeletal conditions as well as visual, vestibular (e.g., vertigo), or sensory (e.g., diabetes) conditions that could negatively affect their postural control. The participants had been informed about the aim and procedure of the study. They were required to provide consent for their inclusion in the study. The study was conducted in accordance with the guidelines provided for in the Declaration of Helsinki and accepted by the institutional Ethics Committee.

\section{Experimental approach to the problem}

This was a randomised controlled trial that aimed to verify whether a short-term, intensive neuromuscular training programme would improve the postural stability of professional soccer players. The experimental group actively participated in an intense, 3-week training programme during the preparatory period, which was completed in addition to their normal scheduled training practice. Later, they exercised 2 times per week for 5 months during in-season maintenance training. The control group were not given any additional training. All of the subjects underwent a postural stability evaluation at the beginning of the study, then after 3 weeks of the programme, and finally 5 months into the programme.

Table 1. The descriptive characteristics of the experimental and control groups (mean $\pm \mathrm{SD}$, max, and $\min$ )

\begin{tabular}{|c|c|c|}
\hline Variable & $\begin{array}{c}\text { Experimental group } \\
(\mathbf{n}=16)\end{array}$ & $\begin{array}{c}\text { Control group } \\
(\mathbf{n}=\mathbf{2 0})\end{array}$ \\
\hline Age (years) & $\begin{array}{c}24.0 \pm 5.0 \\
18.0 \div 37.0\end{array}$ & $\begin{array}{c}22.0 \pm 4.6 \\
18.0 \div 29.0\end{array}$ \\
\hline Body height (cm) & $182.0 \pm 8.1$ & $179.0 \pm 7.0$ \\
& $171.0 \div 196.0$ & $174.0 \div 189.0$ \\
\hline & $77.2 \pm 7.4$ & $75.6 \pm 6.2$ \\
Body mass (kg) & $65.8 \div 91.3$ & $62.1 \div 85.5$ \\
\hline $\begin{array}{c}\text { Training experience } \\
\text { (years) }\end{array}$ & $12.0 \pm 2.4$ & $11.0 \pm 3.5$ \\
& $8.0 \div 15.0$ & $7.0 \div 13.0$ \\
\hline
\end{tabular}

\section{Procedures}

Biomechanical measurements were taken using the $\mathrm{HUR}^{\circledR}$ force platform (Finland) and the accompanying software for static stability measurements. The platform has 4 tensometric force sensors that can be used to measure the position of the centre of pressure (COP) movements in the coordinate system associated with the platform. The study took into account the total COP sway path that the centre of pressure of the foot made on the ground during a 30-second trial for each leg. The players' postural stability was evaluated in a one-leg stance, first on the dominant and then on the non-dominant leg. It was measured in the following manner: one 30-second test for each leg was performed with eyes open (EO), and then one 30-second test was completed for each leg with eyes closed (EC). All the tests were performed in one-leg stance with the knee bent at about 15 degrees and with the other limb in slight flexion. The subjects were instructed to stand barefoot, with their hands at their sides, while keeping their knees apart. They were asked to try not to touch the ground during the testing. The total COP sway path of the 30-second trials was subjected to analysis. Any possible distracting factors were eliminated. During the tests, only one player was examined, and there were no other disturbing factors such as noise. To exclude possible variables stemming from factors such as tiredness due to physical exertion, all the players were tested on the same day, when there was no practice scheduled.

\section{Training protocol}

During the 3-week preparatory period, the experimental group underwent intensive training designed by a team of physiotherapists and a strength and conditioning coach. The training took place twice a day, and each session took 20 minutes. During the first two stages, the exercises were done at the beginning of the practice, after the warm-up. The warm-up included 10 minutes of running and 5 minutes of dynamic stretching of the quadriceps femoris, hamstrings, calf, and gluteal muscles. During the final stage, the training was done after the practice, when the players were tired. Their weariness was introduced on purpose as an additional factor included in the study. The entire protocol, which is shown is Table 2, was divided into three stages. Each stage consisted of 5 main exercises focusing on balance, agility, coordination, neuromuscular control, and plyometric training

Table 2. Training protocol, names of stages (Basic Stability, Advanced Stability, and Advances Dynamic Stability), levels (1-3), duration (20 minutes), and incorporation during training session (level 1 and 2 incorporated after the warm-up, level 3 incorporated after main part of a training session)

\begin{tabular}{|c|c|c|c|}
\hline Name of stage & Level & Duration & $\begin{array}{l}\text { Incorporation } \\
\text { during training } \\
\text { session }\end{array}$ \\
\hline \multirow{2}{*}{ BASIC STABILITY } & $1 \mathrm{~A}$ & $\begin{array}{l}1-3 \text { days, } \\
20 \text { minutes }\end{array}$ & After warm-up \\
\hline & $1 \mathrm{~B}$ & $\begin{array}{l}4-7 \text { days, } \\
20 \text { minutes }\end{array}$ & After warm-up \\
\hline ADVANCED STABILITY & 2 & $\begin{array}{l}8-14 \text { days, } \\
20 \text { minutes }\end{array}$ & After warm-up \\
\hline $\begin{array}{l}\text { ADVANCED DYNAMIC } \\
\text { STABILITY }\end{array}$ & 3 & $\begin{array}{l}15-21 \text { days, } \\
20 \text { minutes }\end{array}$ & $\begin{array}{c}\text { After main part of a } \\
\text { training session } \\
\text { (under fatigue) }\end{array}$ \\
\hline
\end{tabular}

The first stage (Basic Stability) was two-fold. In the first part (Level 1A), which lasted until the 3rd day, the researchers attempted to make the players realise the importance of proper execution of the techniques given. The aim was to make them aware of the impact which keeping the proper axis of the lower limbs and positioning the pelvis has on stability and, subsequently, on performance. All of the exercises in this part of 
the first stage were done on uneven ground while in double-leg stance (Fig. 1). In the second part (Level 1B, from the 4th until the 7 th day of training), the exercises were exactly the same, yet they were performed in the one-leg standing position (Fig. 2). At that point, the supervising coach continually reminded the subjects about the importance of precision while performing each task.

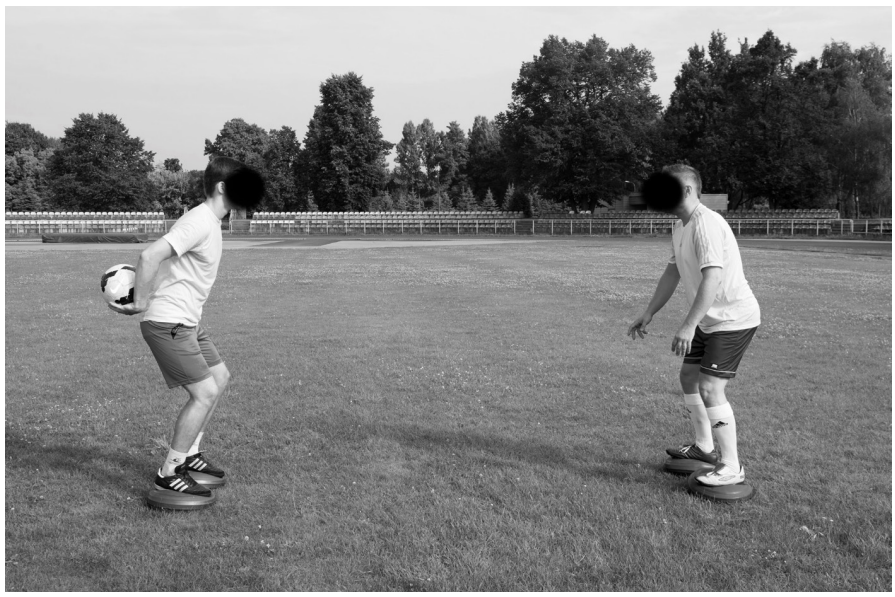

Figure 1. Example of a level 1A exercise (the soccer player keeps his balance while standing on both legs on a balance disk; he catches the ball, circles it around his body, and then throws it back to his partner)

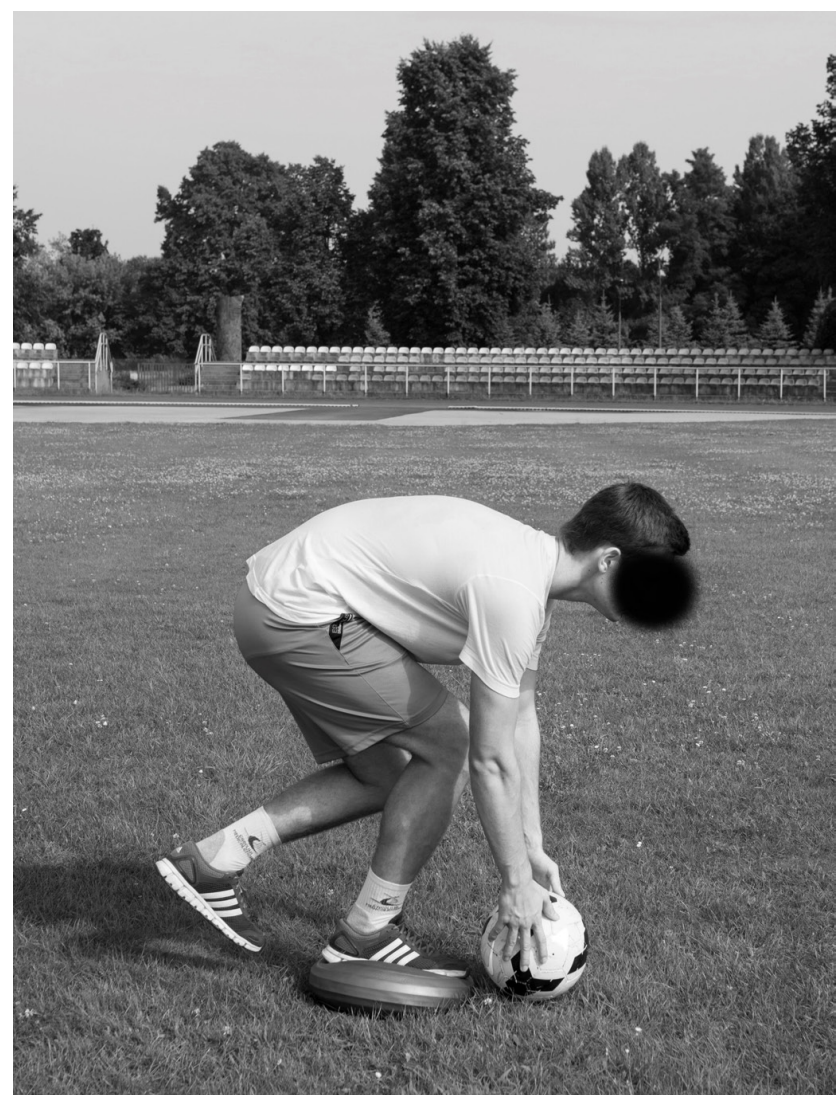

Figure 2. Example of a level 1B exercise (the soccer player keeps his balance while standing on one leg on a balance disk; he catches the ball, performs a squat deep enough for the ball to touch the ground, and at then throws it back to his partner)
The second stage (Advanced Stability, Level 2) took place between the 8th and 14th day and included some dynamic exercises on one and both legs, multidirectional jumps, and jumps with a change in direction during landing, as well as exercises incorporating elements of soccer, such as kicking the ball and heading (Fig. 3). In this stage, the importance of proper landing technique was emphasised.

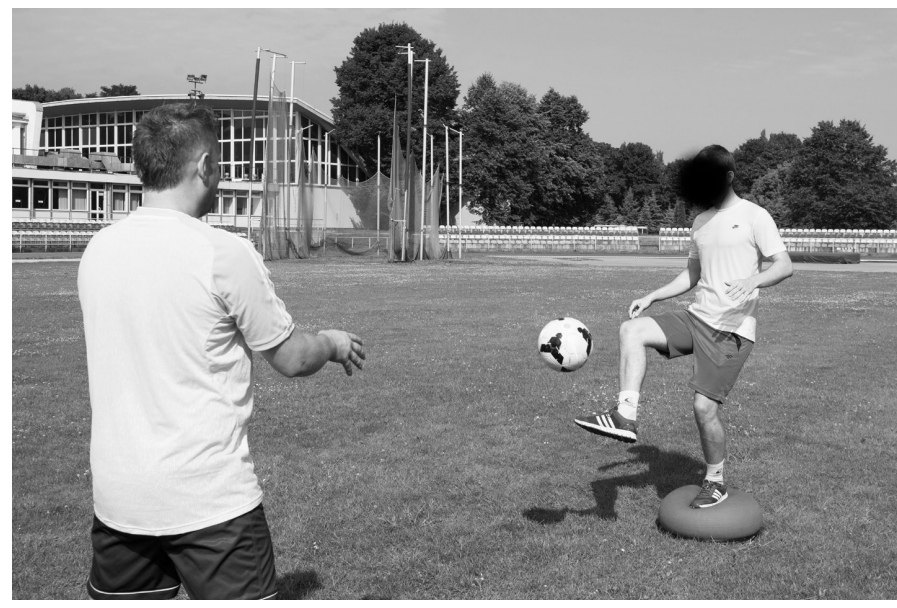

Figure 3. Example of a level 2 exercise (the soccer player jumps from one side onto the TOGU Jumper ${ }^{\circledR}$ (one leg) and then kicks the ball, played by his partner, with the other lower limb)

The final stage (Advanced Dynamic Stability, Level 3) began on the 15th day and lasted until the end of the training period. It consisted of dynamic exercises combined with typical soccer activities, including accelerating and decelerating abruptly. The tasks were complex and involved the use of various equipment, such as balance disks, bosu balls, and a trampoline (Fig. 4). Exercises from levels 2 and 3 included typical discipline-related activities. It was essential to remind the subjects that while they were doing all of the exercises, the dynamic axis of the lower extremity (dynamic Q-angle) had to be kept straight, so that the occurrence of either varus or valgus was controlled and thus limited.

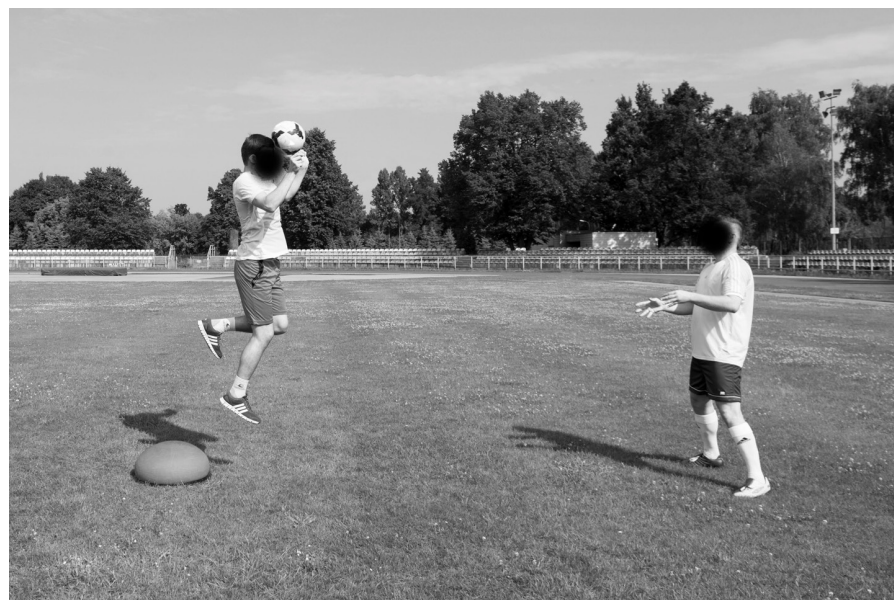

Figure 4. Example of a level 3 exercise (the soccer player jumps onto the TOGU Jumper ${ }^{\circledast}$ on one leg, keeps his balance for a few seconds, performs a vertical jump, heads the ball, lands on one leg, and accelerates) 


\section{Statistical analyses}

In the description of the results, basic statistical values were used: means, standard deviations, and ranges. The assumptions of normality and homogeneity of variances were verified using the Shapiro-Wilk and Levene tests, respectively. Due to certain departures from normality, the data were log-transformed prior to the analysis. The COP data were analysed separately for the eyes open/closed conditions, each time using a three-factor (2 $\times 3 \times 2$ ) mixed design (split-plot) analysis of variance (ANOVA) with one between-subject variable (group) and two withinsubject variables (measurement and $\operatorname{limb}$ ); partial eta-squared $\left(\eta^{2}\right)$ was used as a measure of effect size. The assumption of sphericity was tested with the Mauchly test. Any violations of this assumption were corrected using the Greenhouse-Geisser adjustment. Where appropriate, Tukey's HSD post-hoc tests were used to assess differences between individual means. The level of alpha $=0.05$ was considered significant.

\section{Results}

The analyses performed revealed significant group $\times$ measurement interactions for both variants, that is measurements taken with eyes open $\left(F_{2,68}=12.9 ; \mathrm{p}<0.001 ; \eta^{2}=0.276\right)$ and closed $\left(F_{2,68}=3.18 ; p=0.048 ; \eta^{2}=0.085\right)$. A significantly $(p$ $<0.001$ ) shorter COP sway path, as compared to the initial measurement, was obtained by the experimental group after a 3-week training programme in a measurement taken with eyes open; the COP sway path for the dominant leg was shorter by about $210 \mathrm{~mm}$ and that for the non-dominant leg by about 157 $\mathrm{mm}$. The measurement with eyes closed performed in the experimental group showed that COP sway path was shorter ( $\mathrm{p}<$ 0.05 ) by nearly $660 \mathrm{~mm}$ for the dominant leg and by $530 \mathrm{~mm}$ for the non-dominant leg compared to the initial stage. The training effects in the experimental group were sustained after 5 months, and the results achieved differed significantly from the pre-training ones in measurements taken with eyes open ( $p$ $<0.001)$ as well as with eyes closed $(\mathrm{p}<0.05)$. The COP parameters in the control group did not reveal any improvement after 3 weeks or after 5 months.

No significant interaction for the $\lim b \times$ measurement $\times$ group effect was observed either for eyes open $\left(\mathrm{F}_{2,68}=0.23\right.$; $\mathrm{p}$ $\left.=0.79 ; \eta^{2}=0.007\right)$ or closed $\left(F_{2,68}=1.08 ; p=0.35 ; \eta^{2}=0.031\right)$, which indicates a lack of differences in the level of stability between the dominant and non-dominant leg before and after the training programme in both groups. Additionally, main effects for the limb factor did not prove to be significant in any measurement variant $\left(\mathrm{F}_{1,34}=0.38 ; \mathrm{p}=0.54 ; \eta^{2}=0.011\right.$ and $\mathrm{F}_{1,34}=0.99$; $\mathrm{p}=0.32 ; \eta^{2}=0.028$ for eyes open and closed, respectively). The detailed COP sway path results obtained in the experimental and control groups are presented in Table 3.

\section{Discussion}

The purpose of this study was to determine the effectiveness of a short-term, 3-week intensive neuromuscular training programme on postural stability of professional soccer players and to establish whether postural stability depends on lower extremity dominance. Since there is no standardised standpoint regarding the duration of such programmes, we wished to create a programme that would be more intense yet shorter than most of the existing ones. Statistically significant results in reducing COP path length were achieved after implementing a 3-week training protocol performed twice a day for $20 \mathrm{~min}$ utes as part of a normal training schedule. The results of our
Table 3. Total Centre of Pressure sway path $(\mathrm{mm})$ for the tests with eyes open and closed for the experimental and control groups (mean $\pm \mathrm{SD}$ )

\begin{tabular}{|c|c|c|c|c|}
\hline & \multicolumn{2}{|c|}{$\begin{array}{l}\text { Study group } \\
(n=16)\end{array}$} & \multicolumn{2}{|c|}{$\begin{array}{l}\text { Control group } \\
(n=20)\end{array}$} \\
\hline & $\mathrm{DL}$ & NDL & $\mathrm{DL}$ & NDL \\
\hline \multicolumn{5}{|c|}{ Eyes open } \\
\hline Pre-test & $1443 \pm 299$ & $1373 \pm 324$ & $1370 \pm 250$ & $1345 \pm 241$ \\
\hline $\begin{array}{l}\text { Post-test, } \\
3 \text { weeks }\end{array}$ & $1232 \pm 224^{* * *}$ & $1216 \pm 313^{* * *}$ & $1424 \pm 261$ & $1397 \pm 221$ \\
\hline $\begin{array}{l}\text { Post-test, } \\
5 \text { months }\end{array}$ & $1181 \pm 165^{\star \star \star}$ & $1162 \pm 157^{* * *}$ & $1364 \pm 233$ & $1373 \pm 175$ \\
\hline \multicolumn{5}{|c|}{ Eyes closed } \\
\hline Pre-test & $3558 \pm 1265$ & $3603 \pm 1813$ & $3448 \pm 743$ & $3578 \pm 714$ \\
\hline $\begin{array}{l}\text { Post-test, } \\
3 \text { weeks }\end{array}$ & $2899 \pm 944^{*}$ & $3073 \pm 1028^{*}$ & $3464 \pm 718$ & $3509 \pm 682$ \\
\hline $\begin{array}{l}\text { Post-test, } \\
5 \text { months }\end{array}$ & $2891 \pm 761^{*}$ & $3213 \pm 1161^{*}$ & $3366 \pm 617$ & $3412 \pm 711$ \\
\hline
\end{tabular}

DL - dominant leg; NDL - non-dominant leg; significantly different from respective pretest value: ${ }^{*}-\mathrm{p}<0.05 ;{ }^{* *}-\mathrm{p}<0.001$.

study also showed that the maintenance training performed inseason (a 20-minute session organised biweekly) allowed the effects of the main training period on players' performance to be extended. However, the results of this study did not reveal any statistically significant differences between the dominant and non-dominant leg.

There are many papers describing positive effects of neuromuscular training on soccer skills and stressing that it is successful in both boosting players' abilities and in preventing injuries. Based on the studies published, it cannot be objectively stated how prevention programmes can be optimised to make them time-efficient and which exercises should be included: a selection of the existing ones or possibly a set of completely new ones designed with the sole purpose of such training in mind [23]. Most current prevention programmes last 6 to 8 weeks and are proven to be effective [7]. In their paper, Zech et al. [7] report that programmes lasting 6 weeks and longer have had greater effects on postural sway than four-week programmes. However, the results of our research as well as the findings of Carafa et al. [13] show that a high intensity of the training and a short duration of the programme can quickly improve a player's stability parameters. In addition, the use of maintenance training during the season has also yielded positive results in sustaining the results achieved.

Most authors describe neuromuscular training only as balance and stabilisation exercise, while others define it as a multi-intervention programme $[25,26]$. However, the literature indicates that the most effective programmes are multicomponential [6], which is why the authors of this programme introduced sport-specific exercises focusing on stability, agility, coordination, and plyometrics and included a number of varied activities constantly challenging the players' balance [24]. Nevertheless, in order to create a more comprehensive and multifaceted preventive programme, we should implement musclestrength exercises or static and dynamic stretching exercises. Such elements are included in the best known current injury 
prevention protocol focusing on soccer - the FIFA 1l+ warmup programme $[27,28]$. It contains exercises meant to improve lower-limb and core strength, as well as neuromuscular control, under static and dynamic conditions. Our programme, however, focuses mainly on sport-specific exercises and includes soccer elements, such as kicking, changing direction while running and landing, accelerating and decelerating abruptly, or heading. In this programme, players train under varied conditions (different kinds of unstable ground and activity types). Unstable ground allows for constant stimulation of the player's neurological system as well as their entire body. Avoiding routine was our intention. Moreover, psychological motivation was used (a ball was included in the training), as a soccer player would rather play with a ball than exercise without it. FIFA 1l+ does not include activities with a ball or exercising on uneven ground; hence, the exercise conditions are far removed from the reality of the game.

Another important issue for the player is the scheduling of a neuromuscular programme. The main questions which need to be answered are whether it is better to implement it in the preparatory period or during the season and as part of a training session or at a different time. Carafa et al. [13] describe a programme in many respects similar to the one designed by the authors of this article. In their case, the programme was completed during the pre-season preparatory period, which lasted 30 days (4 weeks), and was performed daily for 20 minutes. It comprised 5 progressive phases, including proprioception training with balance boards. The main training period was followed by an in-season maintenance programme, which involved training 3 times a week, including performing lower extremity proprioception and dynamic balance exercises. The authors of the current paper also proposed a programme implemented during the preparatory period, twice a day for 3 weeks, and an in-season maintenance programme, in which training was done twice a week for 20 minutes. Our programme included 3 stages; stages 1 and 2 were part of the warm-up, which was the same as in the FIFA 11+ warm-up programme. As it is indicated in the name, the FIFA programme is designed to be performed at the beginning of the practice session. In our programme, the last stage was implemented after the main part of the training, when the players were tired. As epidemiological data indicate, many injuries of the lower extremity occur at the end of the first half and at the very end of a match [29], when players are most fatigued. Therefore, in order to reflect the conditions during a match, a shift in the training session schedule was introduced during the course of the training period - the most intense stage (level 3 ) of the protocol was performed after the practice, considering the previously mentioned epidemiological fact that most injuries occur in the second half of a match.

It has been reported that non-contact injuries constitute about 39\% of all injuries [30]. Common on-the-pitch situations which often result in an injury of the knee and ankle include changing direction and/or cutting manoeuvres frequently combined with accelerating and decelerating, landing from a jump in or near full extension, and pivoting [30]. Taking these epidemiological data into account, when creating our neuromuscular programme, we focused on important training elements such as performing exercises in a closed kinematic chain, where the focus is directed at knee and pelvis control, quick changes of direction, as well as accelerations and decelerations. We paid special attention to one of the riskiest moments while playing soccer, which is the landing [6]. Due to its critical importance, the programme needed to teach the techniques of its performance, such as soft landing with initial forefoot contact combined with hip and knee flexion, and ways of controlling the lower limb axis $[31,32]$. It is extremely important not only to introduce appropriate exercises but also to constantly monitor the quality of the exercise performed.

An interesting notion is that data in the literature indicate that soccer players' level of competition correlates with postural stability [4, 33, 34]. Moreover, Paillard et al. [35] suggest that soccer players are able to shift their proprioception so that it does not rely predominantly on sight, but on the receptors of the locomotor system, thus minimising the role of vision. This means that the more proficient the player is, the less they will focus on observing the ball, and the more they will focus on the surroundings and other players to whom they may potentially play the ball. The authors explain that such adaptations are due to rapid changes of the conditions on the field, resulting in players using their vision mostly to assess and react to events occurring on the pitch, that is other players' positions and movements. The results presented in this article indicate that players did show improvement of postural control during tests with their eyes closed, but more improvement was noticed in open-eye tests. This suggests better proprioception and adaptive capabilities to environmental changes with visual stimuli and may indicate that the players who were tested did not have the highest skill level.

A proficient soccer player should be able to kick the ball well with either leg, even though most of them show bilateral differences. When it comes to penalty kicks, in most cases, players choose their preferred leg to shoot. Still, it is controversial whether it is the dominant leg the player chooses for a penalty kick. At the beginning of the study, the authors hypothesised that the dominant leg is the one chosen when taking a penalty, which in turn necessitates that the supporting limb be more stable. However, the results of this study and the results of other authors did not reveal any statistically significant differences between the dominant and non-dominant leg [36-39]. In light of these facts, there is no need to differentiate between the dominant and non-dominant legs when implementing a neuromuscular programme.

\section{Limitations}

One of the shortcomings of this study might be the limited number of subjects who completed all three stages of the research. However, the group tested consisted of professional soccer players who had to complete a 5-month training programme, and many of them dropped out during the course of the programme due to their inability to fully participate in it, changing teams, or injury.

It may also be assumed that the equipment required for our programme, in the form of physiotherapy devices, may be problematic and discouraging for coaches. We believe, however, that the kind of equipment we used in the exercises proposed should be available at any soccer club and be used for both prevention purposes and as an aid when returning to play. Moreover, if a more comprehensive neuromuscular programme is to be offered, it needs to include exercises aimed at improving muscle strength and static and dynamic stretching.

\section{Conclusions}

The following conclusions were drawn from the study:

1. A 3-week training programme applied during the preparatory period seems to be an effective tool for achieving rapid improvement in the postural stability of professional soccer players. 
2. Implementing the programme twice a week during the season allowed the achieved effects on postural stability to be maintained.

3. A combination of balance, coordination, agility, plyometric, and core control exercises appears to be a successful training protocol for improving postural control.

4. Since between-measurement improvements of postural stability were comparable for both limbs, it does not seem necessary to differentiate between the dominant and non-dominant lower limbs during the implementation of a 3-week intensive neuromuscular training programme.

5. It is highly recommended to use the training programme in everyday practice in soccer.

\section{Acknowledgements}

The authors would like to thank Carolina Medical Center, Warsaw, Poland for making it possible to perform tests at the Functional Diagnostics Center as well as Marcin Adasiak for providing the images for figures showing the exercises performed in the programme. The study was carried out at the Functional Diagnostics Center in Carolina Medical Center, Warsaw, Poland and was financed from our own resources.

\section{Literature}

1. Paillard T., Noé F., Rivière T., Marion V., Montoya R., Dupui P. (2006). Postural performance and strategy in the unipedal stance of soccer players at different levels of competition. Journal of Athletic Training 41(2), 172-176.

2. Butler R.J., Southers C., Gorman P.P., Kiesel K.B., Plisky P.J. (2012). Differences in soccer players' dynamic balance across levels of competition. Journal of Athletic Training 47(6), 616-620. DOI: 10.4085/1062-6050-47.5.14.

3. Era P., Konttinen N., Mehto P., Saarela P., Lyytinen H. (1996). Postural stability and skilled performance - A study on top-level and naive rifle shooters. Journal of Biomechanics 29(3), 301-306.

4. Noe F., Paillard T. (2005). Is postural control affected by expertise in alpine skiing? British Journal of Sports Medicine 39(11), 835-837. DOI: 10.1136/bjsm.2005.018127.

5. Vuillerme N., Danion F., Marin L., Boyadjian A., Prieur J.M., Weise I., et al. (2001). The effect of expertise in gymnastics on postural control. Neuroscience Letters 303(2), 83-86.

6. Alentorn-Geli E., Myer G.D., Silvers H.J., Samitier G., Romero D., Lázaro-Haro C., et al. (2009). Prevention of noncontact anterior cruciate ligament injuries in soccer players. Part 2: A review of prevention programs aimed to modify risk factors and to reduce injury rates. Knee Surgery, Sports Traumatology, Arthroscopy 17(8), 859-879. DOI: 10.1007/ s00167-009-0823-z.

7. Zech A., Hübscher M., Vogt L., Banzer W., Hänsel F., Pfeifer K. (2010). Balance training for neuromuscular control and performance enhancement: A systematic review. Journal of Athletic Training 45(4), 392-403. DOI: 10.4085/1062-605045.4.392.

8. Hewett T.E., Ford K.R., Myer G.D. (2006). Anterior cruciate ligament injuries in female athletes: Part 2, a meta-analysis of neuromuscular interventions aimed at injury prevention. American Journal of Sports Medicine 34(3), 490-498. DOI: $10.1177 / 0363546505282619$.

9. Engebretsen A.H., Myklebust G., Holme I., Engebretsen L., Bahr R. (2008). Prevention of injuries among male soccer players: A prospective, randomized intervention study targeting players with previous injuries or reduced function. The American Journal of Sports Medicine 36(6), 1052-1060. DOI: $10.1177 / 0363546508314432$.

10. Mohammadi F. (2007). Comparison of 3 preventive methods to reduce the recurrence of ankle inversion sprains in male soccer players. The American Journal of Sports Medicine 35(6), 922-926. DOI: 10.1177/0363546507299259

11. Ergen E., Ulkar B. (2008). Proprioception and ankle injuries in soccer. Clinics in Sports Medicine 27(1), 195-217. DOI: 10.1016/j.csm.2007.10.002.

12. Kraemer R., Knobloch K. (2009). A soccer-specific balance training program for hamstring muscle and patellar and Achilles tendon injuries: An intervention study in premier league female soccer. The American Journal of Sports Medicine 37(7), 1384-1393. DOI: 10.1177/0363546509333012.

13. Caraffa A., Cerulli G., Projetti M., Aisa G., Rizzo A. (1996). Prevention of anterior cruciate ligament injuries in soccer. A prospective controlled study of proprioceptive training. Knee Surgery, Sports Traumatology, Arthroscopy 4(1), 19-21.

14. Gilchrist J., Mandelbaum B.R., Melancon H., Ryan G.W., Silvers H. J., Griffin L.Y., et al. (2008). A randomized controlled trial to prevent noncontact anterior cruciate ligament injury in female collegiate soccer players. The American Journal of Sports Medicine 36(8), 1476-1483. DOI: $10.1177 / 0363546508318188$.

15. Croisier J.L., Ganteaume S., Binet J., Genty M., Ferret J.M. (2008). Strength imbalances and prevention of hamstring injury in professional soccer players: A prospective study. The American Journal of Sports Medicine 36(8), 1469-1475. DOI: $10.1177 / 0363546508316764$.

16. Arnason A., Andersen T.E., Holme I., Engebretsen L., Bahr R. (2008). Prevention of hamstring strains in elite soccer: An intervention study. Scandinavian Journal of Medicine E Science in Sports 18(1), 40-48. DOI: 10.1111/j.16000838.2006.00634.x.

17. Gabbe B.J., Branson R., Bennell K.L. (2006). A pilot randomised controlled trial of eccentric exercise to prevent hamstring injuries in community-level Australian football. Journal of Science and Medicine in Sport 9(1-2), 103-109. DOI: 10.1016/j.jsams.2006.02.001.

18. Emery C.A., Cassidy J.D., Klassen T.P., Rosychuk R.J., Rowe B.H. (2005). Effectiveness of a home-based balance-training program in reducing sports-related injuries among healthy adolescents: A cluster randomized controlled trial. Canadian Medical Association Journal 172(6), 749-754. DOI: 10.1503/cmaj.1040805.

19. McGuine T. (2006). Sports injuries in high school athletes: A review of injury-risk and injury-prevention research. Clinical Journal of Sport Medicine 16(6), 488-499. DOI: 10.1097/01.jsm.0000248848.62368.43.

20. McKeon P.O., Hertel J. (2008). Systematic review of postural control and lateral ankle instability, part I: Can deficits be detected with instrumented testing. Journal of Athletic Training 43(3), 293-304. DOI: 10.4085/1062-6050-43.3.293.

21. Hewett T.E., Paterno M.V., Myer G.D. (2002). Strategies for enhancing proprioception and neuromuscular control of the knee. Clinical Orthopaedics and Related Research 402, 76-94.

22. Grooms D.R., Palmer T., Onate J.A., Myer G.D., Grindstaff T. (2013). Soccer-specific warm-up and lower extremity injury rates in collegiate male soccer players. Journal of Athletic Training 48(6), 782-789. DOI: 10.4085/1062-605048.4.08. 
23. Myklebust G., Steffen K. (2009). Prevention of ACL injuries: How, when and who? Knee Surgery, Sports Traumatology, Arthroscopy 17(8), 857-858. DOI: 10.1007/s12178-0179416-5.

24. Bieć E., Kuczyński M. (2010). Postural control in 13-yearold soccer players. European Journal of Applied Physiology 110(4), 703-708. DOI: 10.1007/s00421-010-1551-2.

25. Coughlan G., Caulfield B. (2007). A 4-week neuromuscular training program and gait patterns at the ankle joint. Journal of Athletic Training 42(1), 51-59.

26. Pánics G., Tállay A., Pavlik A., Berkes I. (2008). Effect of proprioception training on knee joint position sense in female team handball players. British Journal of Sports Medicine 42(6), 472-476. DOI: 10.1136/bjsm.2008.046516.

27. Soligard T., Myklebust G., Steffen K., Holme I., Silvers H., Bizzini M., et al. (2008). Comprehensive warm-up programme to prevent injuries in young female footballers: Cluster randomised controlled trial. BMJ 337, 2469. DOI: 10.1136/bmj.a2469.

28. Soligard T., Nilstad A., Steffen K., Myklebust G., Holme I., Dvorak J., et al. (2010). Compliance with a comprehensive warm-up programme to prevent injuries in youth football. British Journal of Sports Medicine 44(11), 787-793. DOI:10.1136/bjsm.2009.070672.

29. Woods C., Hawkins R., Hulse M., Hodson A. (2003). The Football Association Medical Research Programme: An audit of injuries in professional football: An analysis of ankle sprains. British Journal of Sports Medicine 37(3), 233-238. DOI: 10.1136/bjsm.37.3.233.

30. Alentorn-Geli E., Myer G.D., Silvers H.J., Samitier G., Romero D., Lázaro-Haro C., et al. (2009). Prevention of noncontact anterior cruciate ligament injuries in soccer players. Part 1: Mechanisms of injury and underlying risk factors. Knee Surgery, Sports Traumatology, Arthroscopy 17(7), 705-729. DOI: 10.1007/s00167-009-0813-1.
31. Quammen D., Cortes N., Van Lunen B.L., Lucci S., Ringleb S.I., Onate J. (2012). Two different fatigue protocols and lower extremity motion patterns during a stop-jump task. Journal of Athletic Training 47(1), 32-41.

32. Blackburn J.T., Padua D.A. (2008). Influence of trunk flexion on hip and knee joint kinematics during a controlled drop landing. Clinical Biomechanics 23(3), 313-319. DOI:10.1016/j.clinbiomech.2007.10.003.

33. Besier T.F., Lloyd D.G., Cochrane J.L., Ackland T.R. (2001). External loading of the knee joint during running and cutting maneuvers. Medicine and Science in Sports and Exercise 33(7), 1168-1175.

34. Matsuda S., Demura S., Uchiyama M. (2008). Centre of pressure sway characteristics during static one-legged stance of athletes from different sports. Journal of Sports Sciences 26(7), 775-779. DOI: 10.1080/02640410701824099.

35. Paillard T., Bizid R., Dupui P. (2007). Do sensorial manipulations affect subjects differently depending on their postural abilities? British Journal of Sports Medicine 41(7), 435438. DOI: $10.1136 /$ bjsm.2006.032904.

36. Alonso A.C., Brech G.C., Bourquin A.M., Greve J.M.D. (2011). The influence of lower-limb dominance on postural balance. Revista Paulista de Medicina 129(6), 410-413.

37. Raymundo A.C, Pernamuco C.S., Brum R.D., Printo de Casto J.B. (2018) Evaluation of strength, agility and aerobic capacity in Brazilian football players. Biomedical Human Kinetics $10,25-30$.

38. Kiyota T., Fujiwara K. (2014). Dominant side in single-leg stance stability during floor oscillations at various frequencies. Journal of Physiological Anthropology 33(1), 25. DOI: 10.1186/1880-6805-33-25.

39. Riemann B.L., Schmitz R. (2012). The relationship between various modes of single leg postural control assessment. International Journal of Sports Physical Therapy 7(3), 257266.

Submitted: December 12, 2018.

Accepted: March 28, 2019. 